\title{
Dust Induced Dynamics of Circumstellar Shells around Long-Period Variables
}

\author{
A. Gauger, A.J. Fleischer, E. Sedlmayr \\ Institut für Astronomie und Astrophysik, Berlin, Germany
}

\section{Dynamical modelling of circumstellar dust shells around LPVs}

The presence of dust essentially affects the optical appearance and the dynamics of circumstellar shells around LPVs, while in turn the formation of solid particles as well as their properties critically depend on the actual local conditions. Thus, a reliable modelling of the structure and dynamics of such shells requires a detailed treatment of the dust complex.

In our hydrocode, which is described in more detail in Fleischer et al. (1992), the time evolution of the dust component is treated consistently by means of a moment method (Gauger et al. 1990). For the solution of the hydrodynamical and thermodynamical problem we essentially follow the approach of Bowen (1988), except for the shocks, which so far are assumed to be isothermal.

\section{Dust induced dynamical phenomena}

In our models the complex interplay between the dust component and the hydroand thermodynamical structure of the shell leads to (cf. Fleischer et al. 1991, 1992):

1. An inhomogeneous shell-like distribution of the circumstellar dust, caused by the variations of the temperature, the density and the chemical composition in the dust forming region.

2. Pronounced shocks dominating the shell structure, which are either generated by the interior pulsation and amplified substantially by radiation pressure on newly formed dust grains, or even created by radiation pressure on dust alone.

3. A substantial backwarming effect due to the dust opacity, which increases the radiative equilibrium temperature inside the new dust layer by several hundred Kelvins, and thereby determines the inner boundary of the region where dust can be formed.

The resulting implications for observable quantities are currently under investigation.

\section{References:}

Bowen, G.H., 1988, Astrophys.J. 329, 299

Fleischer, A.J., Gauger, A., Sedlmayr, E., 1991, Astron. Astrophys., 242, L1

Fleischer, A.J., Gauger, A., Sedlmayr, E., 1992, Astron. Astrophys., in press

Gauger, A., Gail, H.-P., Sedlmayr, E., 1990, Astron. Astrophys., 235, 345 